\title{
In situ high energy resolution off-resonant spectroscopy applied to a time-resolved study of single site Ta catalyst during oxidation
}

\author{
Wojciech Błachucki ${ }^{\text {a,* }}$, Jakub Szlachetko ${ }^{\text {b,c }}$, Yves Kayser ${ }^{b}$, Jean-Claude Dousse ${ }^{a}$, Joanna Hoszowska ${ }^{\text {a }}$, \\ Faisal Zeeshan ${ }^{\text {a }}$, Jacinto Sád,e \\ ${ }^{a}$ Department of Physics, University of Fribourg, Chemin du Musée 3, CH-1700 Fribourg, Switzerland \\ ${ }^{\mathrm{b}}$ Swiss Light Source, Paul Scherrer Institut, $\mathrm{CH}-5232$ Villigen-PSI, Switzerland \\ ${ }^{c}$ Institute of Physics, Jan Kochanowski University, 25-406 Kielce, Poland \\ ${ }^{\mathrm{d}}$ Department of Chemistry, Uppsala University, 75237 Uppsala, Sweden \\ e Institute of Physical Chemistry, Polish Academy of Sciences, 01-224 Warsaw, Poland
}

\begin{abstract}
In the present work high energy resolution off-resonant X-ray spectroscopy (HEROS) was employed at a synchrotron to study a silica supported $\mathrm{Ta}(\mathrm{V})$ bisalkyl catalyst activated in hydrogen. The Ta $L \alpha_{1}$ HEROS spectra were measured during oxidation of the starting complex and the relative species' concentration was successfully retrieved as a function of time using the fingerprint HEROS spectra measured for the unoxidized and the oxidized catalyst. Based on the experimental data and theory-based calculations, it was shown that oxidation of the active Ta catalyst leads to the formation of mono- and di-meric species on the $\mathrm{SiO}_{2}$ surface. The obtained results were compared to those of the previously reported timeresolved HEROS study on an inactive silica supported $\mathrm{Ta}(\mathrm{V})$ bisalkyl catalyst's concentration evolution during its oxidation Błachucki et al. (2015). The study allowed observation of an immediate transition of the active Ta catalyst from its unoxidized form to the oxidized one. This finding is dissimilar to the result of the study on the inactive Ta catalyst, where the oxidation led through an intermediate step.
\end{abstract}

\section{Introduction}

High energy resolution off-resonant X-ray spectroscopy (HEROS) is a method of determining the density of unoccupied electronic states [1-3]. HEROS is an alternative to the existing X-ray absorption spectroscopy (XAS) methods and opens the way for new studies not achievable before. HEROS makes use of high energy resolution X-ray emission spectroscopy (XES) to study spectra of inelastically scattered photons in off-resonant conditions, i.e., for incident photon energies below the atomic core level binding energy of interest. It has been shown that such spectra carry information on the occupancy of the discrete valence electronic states as well as the states above the ionization threshold (in the continuum) [4-7]. Provided that the incident photon beam energy bandwidth is smaller than the initial state lifetime broadening and a high resolution wavelength-dispersive detection system is used, HEROS allows to probe the density of unoccupied states with high energy resolution. This is possible because in the

\footnotetext{
* Corresponding author.

E-mail address: wojciech.blachucki@unifr.ch (W. Błachucki).
}

off-resonant inelastic X-ray scattering process the scattered photons' spectra are not broadened by the lifetime of the core hole associated to the investigated absorption edge. Further, in HEROS the sample is irradiated with a monochromatic X-ray beam of energy fixed below the given ionization threshold and the crystal spectrometer (in the von Hamos or Johansson geometry), equipped with a position sensitive detector, allows recording emission spectra in one shot with covered energy ranges of typically tens of eV. This scanning-free arrangement thus allows to probe the density of unoccupied states with time resolution limited only by the experimental setup's efficiency. The swift way of probing the electronic structure of matter, provided by HEROS, is highly desirable in timeresolved spectroscopic studies on dynamically changing chemical systems. This also makes HEROS a method of choice in case of extremely bright pulsed X-ray sources such as X-ray free electron lasers (XFELs), where the target is damaged after every pulse and the beam intensity variations severely limit the usability of conventional XAS methods [8]. Moreover, the HEROS spectral profiles are not modified by the self-absorption effect [9].

The ability of the HEROS method to perform time-resolved studies on chemically dynamic systems has already been used to follow the time course of such chemical processes as oxidation, 
reduction and compound decomposition [1,10,11]. It has been shown that, despite the low fluorescence yield of off-resonant excitations, HEROS allows the observation of changes in the density of unoccupied states caused by the evolving chemical environment of the probed atoms with a high temporal resolution reaching $1 \mathrm{~s}$ or less. Moreover, the insensitivity of HEROS to the self-absorption effect [9] makes it a reliable tool to perform quantitative chemical speciations under reaction conditions.

\section{Theory}

As reported by Tulkki and Åberg [5], the photon scattering cross section for the off-resonant excitation well below the ionization threshold is correlated to the density of unoccupied states. Assuming that the fluorescence yield $I_{X E S}$ is proportional to the scattering cross section and the oscillator strength distribution for electron excitation $I_{X A S}$ to the density of unoccupied states, one can reformulate the Tulkki and Åberg formula to retrieve the XAS data from the off-resonant XES data and vice versa [1,6]. In the present work, the density of unoccupied states of Ta atoms is measured through the detection of the photons emitted in the $L_{3} \rightarrow M_{5}$ decay channel in off-resonant conditions. In this case, the Tulkki and Åberg formula has the following form:

$$
\begin{aligned}
I_{X E S}\left(\hbar \omega_{2}\right) \propto & \int_{0}^{\infty}\left[\frac{\hbar \omega_{2}}{\hbar \omega_{1}} \frac{\left(\left|E_{L_{3}}\right|-\left|E_{M_{5}}\right|\right)\left(E+\left|E_{L_{3}}\right|\right)}{\left(E+\left|E_{L_{3}}\right|-\hbar \omega_{1}\right)^{2}+\Gamma_{L_{3}}^{2} / 4}\right. \\
& \times \frac{\Gamma_{M_{5}}}{\left(\hbar \omega_{1}-\hbar \omega_{2}-\left|E_{M_{5}}\right|-E\right)^{2}+\Gamma_{M_{5}}^{2} / 4} \\
& \left.\times I_{X A S}(E)\right] \mathrm{d} E
\end{aligned}
$$

where $E_{L_{3}}$ and $E_{M_{5}}$ represent the electron binding energies in the initial and final states, respectively, and $\hbar \omega_{1}$ and $\hbar \omega_{2}$ stand for the energies of the incident and emitted photons. The initial and final state lifetime broadenings are denoted by $\Gamma_{L_{3}}$ and $\Gamma_{M_{5}}$. The $I_{X A S}(E)$ function is proportional to the linear photoabsorption coefficient $\mu_{\mathrm{ph}}$ as a function of the excitation energy $\left(E+\left|E_{L_{3}}\right|\right)$. The offresonant XES data $I_{X E S}\left(\hbar \omega_{2}\right)$ retrieved from the $I_{X A S}(E)$ function by means of Eq. (1) is proportional to the intensity of emitted photons as a function of their energy. It can be noted first that $I_{X E S}\left(\hbar \omega_{2}\right)=I_{X E S}\left(\hbar \omega_{1}-\left|E_{M_{5}}\right|-E\right)$ according to the energy conservation law for the resonant inelastic X-ray scattering (RIXS) $[5,6]$ and secondly that for incident energies tuned about $15 \mathrm{eV}$ below the $L_{3}$ edge, like in the present experiment, the XES intensity given by Eq. (1) is nearly insensitive to the initial state width $\Gamma_{L_{3}}$.

\section{Samples and experimental method}

The measurements were carried out at the SuperXAS beamline of the Swiss Light Source of the Paul Scherrer Institut, Switzerland. The collimated synchrotron beam was monochromatized by means of a double $\mathrm{Si}(111)$ crystal monochromator and focused with a toroidally bent Rh mirror to a $100 \times 100 \mu \mathrm{m}^{2}$-spot size on the target. First, a Ta foil was used as the target to calibrate in energy the incident beam and the spectrometer. Then the investigated Ta complex was loaded into a quartz capillary reactor cell in a glove box and exposed to $20 \mathrm{ppm} \mathrm{O}_{2}$ at room temperature. Two silica supported Ta complexes were studied, $\mathrm{Ta}(\mathrm{V})$ bisalkyl complex (Complex 1) and Ta hydride (Complex 2), i.e., more specifically:

$$
\begin{gathered}
\text { Complex } 1:\left[\left(\equiv \mathrm{SiO}_{2}\right) \mathrm{Ta}(=\mathrm{CHtBu})\left(\mathrm{CH}_{2} \mathrm{tBu}\right)_{2}\right], \\
\text { Complex } 2:\left[\left(\equiv \mathrm{SiO}_{2}\right) \mathrm{TaH}_{3}\right] .
\end{gathered}
$$

The quartz capillary reactor cell was mounted on the sample holder and connected to a remotely switchable gas system which allowed applying a gas flow through the target on line. Two gases were used: Ar to flush residual gases out of the reactor and $10 \% \mathrm{H}_{2} / \mathrm{He}$ mixture to activate the Ta complex. The temperature of the Ta complex was adjusted with an air blower and a thermometer placed on two sides of the reactor.

The induced fluorescence was detected in the energy range around the Ta $L \alpha_{1}$ emission line $(8.146 \mathrm{keV})$ by means of a wavelength-dispersive von Hamos-type spectrometer consisting of two cylindrically curved segmented-type $\mathrm{Si}(444)$ crystals [12] and a two-dimensional PILATUS $100 \mathrm{~K}$ detector [13]. Two crystals were employed to increase the solid angle of the spectrometer. The latter was operated in the vertical dispersive geometry. The two crystals were oriented so that they cover exactly the same Bragg angular range. However, they were slightly tilted to each other about a vertical axis so that the photons diffracted by the two crystals were detected by two different regions of the PILATUS detector. An acquisition time of $40 \mathrm{~s}$ per image was used. For each time interval of $40 \mathrm{~s}$, the HEROS spectrum was obtained by projecting the sum of the two 2D images onto the dispersion axis of the spectrometer. From the full width at half maximum (FWHM) of a Gaussian fit to the elastic scattering peak a total experimental resolution of $1.6 \mathrm{eV}$ was found. A $30-\mathrm{cm}$-long, helium filled, ionization chamber was placed upstream the target to normalize the detected fluorescence yield as a function of the beam intensity.

\section{Results and discussion}

The Ta $L \alpha_{1}$ HEROS spectra measured at an incident beam energy of $9.863 \mathrm{keV}$ during oxidation of Complex 1 are presented in Fig. 1 (a). The reaction took place at room temperature and it caused an increase in the fluorescence intensity as well as a shift of the detected spectral structure towards lower energies. In the case of Complex 2, the Ta $L \alpha_{1}$ HEROS spectra, which are shown in Fig. 1 (b), were collected at an incident beam energy of $9.867 \mathrm{keV}$. Complex 2 was obtained by activating Complex 1 in hydrogen. This was achieved by exposing Complex 1 to a $10 \% \mathrm{H}_{2} / \mathrm{He}$ gas mixture between $1300 \mathrm{~s}$ and $3200 \mathrm{~s}$ while rising the temperature from $20^{\circ} \mathrm{C}$ to $150^{\circ} \mathrm{C}$. During activation, a slight gradual displacement of the detected spectral structure towards lower energies was observed. Further oxidation of Complex 2 led to a sudden spectral shift and increase in intensity of the emitted fluorescence.

As shown in Fig. 2(a), oxidation of Complex 1 caused a shift of the detected spectral structure towards lower energies by about $1 \mathrm{eV}$ as well as an increase in the fluorescence intensity. It also gave rise to the evolution of another spectral structure on the low energy side of the main peak. Fig. 2(b) presents fingerprint Ta $L \alpha_{1}$ HEROS spectra measured for Complex 2 at three different stages: before the formation of Complex 2 from Complex 1 (through activation of Complex 1 in hydrogen), before reaction of the formed Complex 2 with oxygen and after Complex 2 turned into its oxidized form. During activation, a slight gradual displacement of the detected spectral structure towards lower energies (by about $0.5 \mathrm{eV}$ ) was observed. Further oxidation of Complex 2 led to a sudden spectral shift and an increase in intensity of the emitted fluorescence. The shape of the spectrum recorded for the oxidized Complex 2 reveals formation of another peak situated slightly closer in energy to the main peak as compared to the spectrum recorded for oxidized Complex 1.

The evolution during oxidation of the second peak in the HEROS spectra suggests the formation of $\mathrm{Ta}=$ oxo dimeric surface species with octahedral coordination [14], which is consistent with calculations of Ta $L_{3}$ edge XAS spectra and off-resonant Ta $L \alpha_{1}$ XES spectra for a monomeric and a dimeric Ta species (see Fig. 3 ). The absorption spectra were computed using the FEFF program [15] and the emission spectra were obtained using the KramersHeisenberg formula modified by Tulkki and Åberg [4,5] with the 

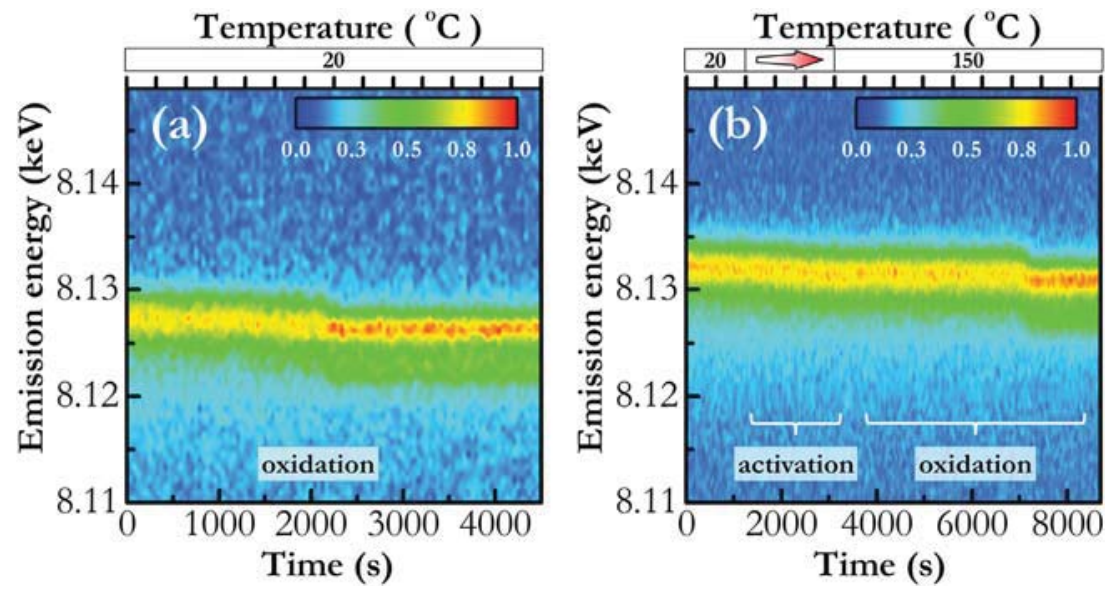

Fig. 1. The measured Ta $L \alpha_{1}$ HEROS spectra as a function of time for Complex 1 (a) and Complex 2 (b). The spectra in (a) and (b) were recorded for an incident beam energy of $9.863 \mathrm{keV}$ and $9.867 \mathrm{keV}$, respectively (i.e., $18 \mathrm{eV}$ and $14 \mathrm{eV}$ below the Ta $L_{3}$ edge, respectively).
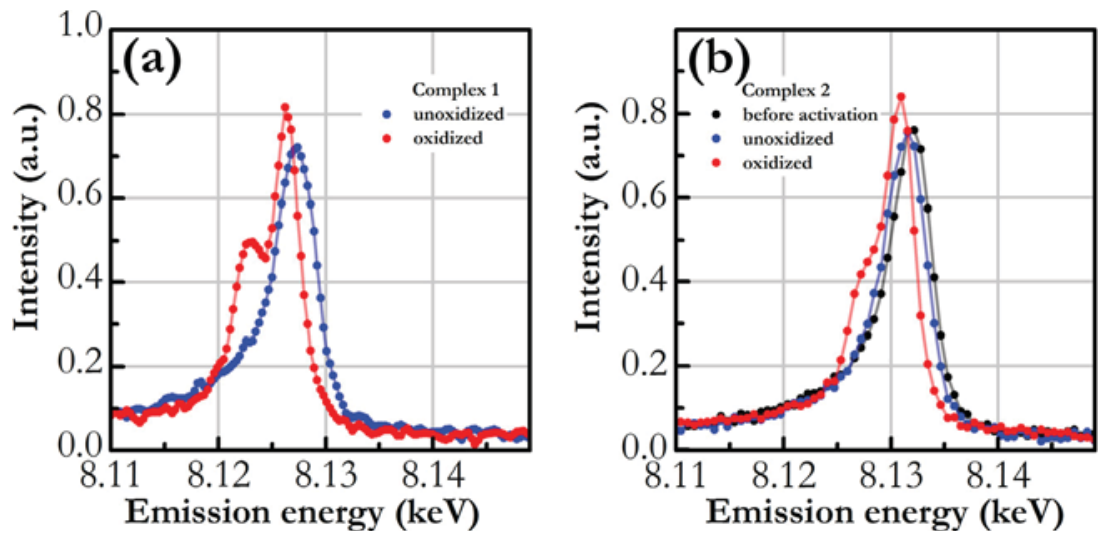

Fig. 2. The fingerprint Ta $L \alpha_{1}$ HEROS spectra measured before and after oxidation of Complex 1 (a) and Complex 2 (b). The plot in (b) also shows the fingerprint HEROS spectrum of Complex 2 before its formation, i.e., before activation of Complex 1 in hydrogen.
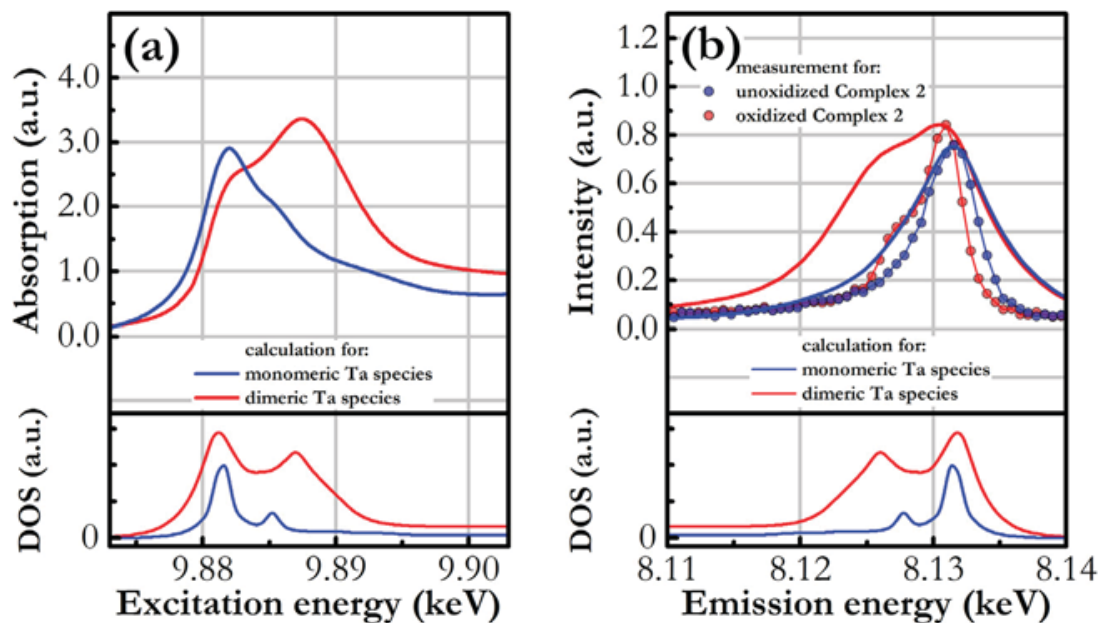

Fig. 3. (a) The Ta $L_{3}$ XANES calculated with FEFF [15] for a monomeric and a dimeric Ta species. The lower panel shows the distribution of the Ta atoms' $d$-states which are contributing the most to the absorption spectra (DOS stands for density of states). (b) Off-resonant Ta $L \alpha_{1}$ emission spectra calculated from the XAS data (a) using Eq. (1). The values for $E_{L_{3}}$ and $E_{M_{5}}$ were taken from Ref. [17] and the values for $\Gamma_{L_{3}}$ and $\Gamma_{M_{5}}$ from Ref. [18]. The data points come from the measurements of the fingerprint HEROS spectra for Complex 2 before and after its oxidation. The lower panel in (b) presents the density of unoccupied states from (a) converted into the emission energy scale using the resonant inelastic X-ray scattering (RIXS) energy conservation law.

result from FEFF as the input data. As it can be seen in Fig. 3(b), the calculated spectra reproduce satisfactorily the overall shapes of the experimental HEROS spectra but theory overestimates the widths of the experimental spectra, in particular for the dimeric species. The observed discrepancies originate probably from the FEFF calculations and not from the values of the parameters used in 

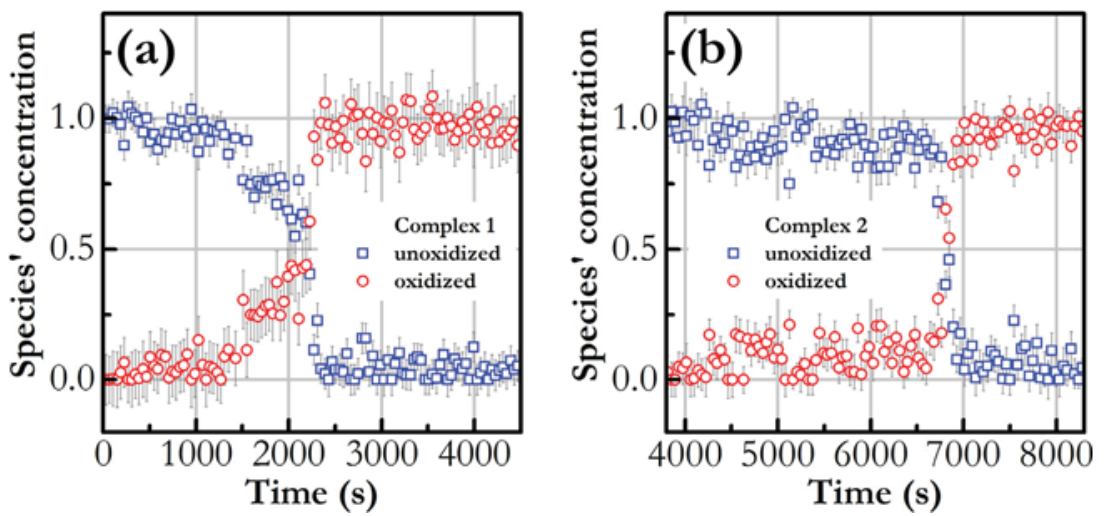

Fig. 4. Relative concentration of Complex 1 [16] (a) and Complex 2 (b) as a function of time during oxidation. The step-wise character of the reaction in the case of Complex 1 was not observed for Complex 2.

Eq. (1) which are all quite accurate (uncertainties of about $1 \mathrm{eV}$ for energies and $0.2 \mathrm{eV}$ for the final state lifetime broadening). The XAS spectra in Fig. 3(a) show that Ta $L_{3}$ XANES is dominated by one peak in the case of monomeric species and by two peaks in the case of dimeric species. The electronic states that contribute most to the absorption spectra are in both cases the $5 d$ orbitals of Ta atoms. As shown in Fig. 3(b), there is a significant difference in the shape of the off-resonant XES spectra calculated for monomeric and dimeric Ta species, and it resides mainly in the presence of a doublet in the case of the latter. The performed calculations thus confirm that the evolution of the second peak in the Ta $L \alpha_{1}$ HEROS spectra measured during oxidation of Complex 1 and Complex 2 is caused by formation of dimeric Ta species. However, the ratio of the two peaks' intensity is different for the spectrum calculated for a dimeric Ta species and the one measured for the oxidized Complex 2 and Complex 1 [16]. This result suggests that both monomeric and dimeric Ta species are present in the samples after oxidation.

For both studied complexes the fingerprint Ta $L \alpha_{1}$ HEROS spectra were recorded before and after oxidation to determine temporal changes in the species' concentration. As can be seen in Fig. 4 (a), during reaction of Complex 1 with oxygen only about $20 \%$ of the sample first turned into its oxidized form and the remaining $80 \%$ transformed afterwards. In the light of the apparent formation of dimeric Ta species during oxidation, one may suppose that the demonstrated step-wise transition is an effect of different reaction rates for oxidation and dimerization. Such a behavior was not observed in the case of Complex 2 oxidation, as shown in Fig. 4 (b), where at about $6800 \mathrm{~s}$ all the sample was suddenly oxidized.

\section{Conclusions}

In the present work high energy resolution off-resonant spectroscopy (HEROS) was used at a synchrotron to study temporal changes in the density of unoccupied states of Ta atoms in silica supported catalysts during oxidation. The catalysts under study were: inactive $\mathrm{Ta}(\mathrm{V})$ bisalkyl catalyst and $\mathrm{Ta}(\mathrm{V})$ bisalkyl catalyst activated in hydrogen. The active site in catalysis, postulated to be Ta hydride, was found to be very susceptible to oxygen and its detection was not reported in any earlier work. Analysis of Ta $L \alpha_{1}$ HEROS spectra measured with 40 s-time resolution showed that oxidation of both catalysts (the inactive one and the active one) leads to the formation of monomeric and dimeric Ta surface species. The catalysis was found to be irreversible. Calculations done with FEFF software [15] and the Kramers-Heisenberg formula modified by Tulkki and Åberg [4,5] revealed that the electronic states contributing most to the measured HEROS spectra come from the Ta $d$-band. The present work is therefore the first to depict the electronic structure of the $d$-orbitals (directly involved in the catalysis) of Ta grafted catalysts. Finally, the evolution of the active Ta catalyst's concentration during oxidation was extracted from the measured HEROS spectra and was compared to the previously reported data from a time-resolved study with HEROS following oxidation of an inactive silica supported $\mathrm{Ta}(\mathrm{V})$ bisalkyl catalyst [16]. The comparison showed that the oxidation of the active Ta catalyst occurs suddenly, which is different from the stepwise evolution of the inactive Ta catalyst oxidation.

\section{Acknowledgements}

We thank the Swiss Light Source at the Paul Scherrer Institut, Switzerland, for access to the SuperXAS beam line and laboratories. Four of us (W. B., J.-Cl. D., J. H. and F. Z.) acknowledge the financial support from the Swiss National Science Foundation. The authors also wish to thank Dr. Szeto, Dr. Mallmann and Dr. Taoufik from CNRS-LCOMS, Université Lyon 1, for preparing the samples and for valuable discussions.

The present work is described in more detail in the Ph.D. thesis of the 1st author [19]. Most of the text contained in the Introduction of the present paper was taken directly from the thesis' abstract and Chapter IV.1, the text contained in the Sect. Samples and experimental method from Chapter IV.2.1 and part of the text in the Sect. Results and discussion from Chapter IV.4.

\section{References}

[1] J. Szlachetko et al., High energy resolution off-resonant spectroscopy at subsecond time resolution: $\left(\mathrm{Pt}(\mathrm{acac})_{2}\right.$ ) decomposition, in: Chem. Commun. 48 (2012) 10898, http://dx.doi.org/10.1039/C2CC35086F.

[2] M. Kavčič, M. Žitnik, K. Bučar, A. Mihelič, B. Marolt, J. Szlachetko, P. Glatzel, K. Kvashnina, Hard X-ray absorption spectroscopy for pulsed sources, Phys. Rev. B 87 (2013) 075106, http://dx.doi.org/10.1103/PhysRevB.87.075106.

[3] J. Sá (Ed.), High-resolution XAS/XES: analyzing electronic structures of catalysts, CRC Press, 2014, ISBN 978-1-4665-9298-8, Techniques: RXES, HRXAS, HEROS, GIXRF, and GEXRF.

[4] H.A. Kramers, W. Heisenberg, Über die Streuung von Strahlung durch Atome, Zeitschrift für Physik 31 (1925) 681, http://dx.doi.org/10.1007/BF02980624.

[5] J. Tulkki, T. Åberg, Behaviour of Raman resonance scattering across the K X-ray absorption edge, J. Phys. B: At. Mol. Phys. 15 (1982) L435, http://dx.doi.org/ 10.1088/0022-3700/15/13/004.

[6] H. Hayashi, R. Takeda, Y. Udagawa, T. Nakamura, H. Miyagawa, H. Shoji, S. Nanao, N. Kawamura, Lifetime-broadening-suppressed/free XANES spectroscopy by high-resolution resonant inelastic X-ray scattering, Phys. Rev. B 68 (2003) 045122, http://dx.doi.org/10.1103/PhysRevB.68.045122.

[7] H. Hayashi, R. Takeda, Y. Udagawa, T. Nakamura, H. Miyagawa, H. Shoji, S. Nanao, N. Kawamura, Lifetime-broadening-removed XANES spectroscopy by high-resolution resonant inelastic X-ray scattering, Phys. Scr. T115 (2005) 1094, http://dx.doi.org/10.1238/Physica.Topical.115a01094.

[8] J. Szlachetko et al., The electronic structure of matter probed with a single femtosecond hard X-ray pulse, Struct. Dyn. 1 (2014) 021101, http://dx.doi.org/ $10.1063 / 1.4868260$ 
[9] W. Błachucki, J. Szlachetko, J. Hoszowska, J.-Cl. Dousse, Y. Kayser, M Nachtegaal, J. Sá, High energy resolution off-resonant spectroscopy for X-ray absorption spectra free of self-absorption effects, Phys. Rev. Lett. 112 (2014) 173003, http://dx.doi.org/10.1103/PhysRevLett.112.173003.

[10] J. Szlachetko, D. Ferri, V. Marchionni, A. Kambolis, O.V. Safonova, C.J. Milne, O. Kröcher, M. Nachtegaal, J. Sá, Subsecond and in situ chemical speciation of Pt/ $\mathrm{Al}_{2} \mathrm{O}_{3}$ during oxidation-reduction cycles monitored by high-energy resolution off-resonant X-ray spectroscopy, J. Am. Chem. Soc. 135 (2013) 19071, http:// dx.doi.org/10.1021/ja410146c.

[11] J. Szlachetko, J. Sá, M. Nachtegaal, U. Hartfelder, J.-Cl. Dousse, J. Hoszowska, D. L.A. Fernandes, H. Shi, C. Stampfl, Real time determination of the electronic structure of unstable reaction intermediates during $\mathrm{Au}_{2} \mathrm{O}_{3}$ reduction, J. Phys. Chem. Lett. 5 (2014) 80, http://dx.doi.org/10.1021/jz402309s.

[12] J. Szlachetko et al., A von Hamos X-ray spectrometer based on a segmentedtype diffraction crystal for single-shot X-ray emission spectroscopy and timeresolved resonant inelastic X-ray scattering studies, Rev. Sci. Instrum. 83 (2012) 103105, http://dx.doi.org/10.1063/1.4756691.

[13] Ch. Broennimann et al., The PILATUS 1M detector, J. Synchrotron Radiat. 13 (2006) 120, http://dx.doi.org/10.1107/S0909049505038665.
[14] R.N. Kapoor, F. Cervantes-Lee, C.F. Campana, C. Haltiwanger, K. Abney, K.H. Pannell, Synthesis, structural and spectroscopic characterization, catalytic properties, and thermal transformations of new cyclic Di- and trisiloxanediolato tantalum complexes, Inorg. Chem. 45 (2006) 2203, http:// dx.doi.org/10.1021/ic051274w.

[15] J.J. Rehr, J.J. Kas, F.D. Vila, M.P. Prange, K. Jorissen, Parameter-free calculations of X-ray spectra with FEFF9, Phys. Chem. Chem. Phys. 12 (2010) 5503, http:// dx.doi.org/10.1039/B926434E.

[16] W. Błachucki, J. Szlachetko, Y. Kayser, J.-Cl. Dousse, J. Hoszowska, D.L.A. Fernandes, J. Sá, Study of the reactivity of silica supported tantalum catalysts with oxygen followed by in situ HEROS, Phys. Chem. Chem. Phys. 17 (2015) 18262, http://dx.doi.org/10.1039/c5cp02950c.

[17] J.A. Bearden, A.F. Burr, Reevaluation of X-Ray Atomic Energy Levels, Rev. Mod. Phys. 39 (1967) 125, http://dx.doi.org/10.1103/RevModPhys.39.125.

[18] J.L. Campbell, T. Papp, WIDTHS OF THE ATOMIC K - N7 LEVELS, At. Data Nucl. Data Tables 77 (2001) 1, http://dx.doi.org/10.1006/adnd.2000.0848.

[19] W. Błachucki, High energy resolution off-resonant X-ray spectroscopy, Faculty of Science of the University of Fribourg, Switzerland, 2015, Ph.D. thesis Nr 1932 (unpublished). 